\title{
Nanoscale
}

PAPER
View Article Online

View Journal I View Issue
Check for updates

Cite this: Nanoscale, 2019, 11, 14070

\section{Microlens array enhanced upconversion luminescence at low excitation irradiance $\uparrow$}

\author{
Qingyun Liu, ${ }^{\mathrm{a}, \mathrm{b}}$ Haichun Liu, (D) *a Deyang Li, ${ }^{\mathrm{b}}$ Wen Qiao, ${ }^{\mathrm{c}}$ Guanying Chen (D) *b and \\ Hans Ågren (D)*a,b,d
}

Received 11th April 2019,

Accepted 22nd June 2019

DOI: $10.1039 / c 9 n r 03105 \mathrm{~g}$

rsc.li/nanoscale

\begin{abstract}
The dearth of high upconversion luminescence (UCL) intensity at low excitation irradiance hinders the prevalent application of lanthanide-doped upconversion nanoparticles (UCNPs) in many fields ranging from optical bioimaging to photovoltaics. In this work, we propose to use microlens arrays (MLAs) as spatial light modulators to manipulate the distribution of excitation light fields in order to increase UCL, taking advantage of its nonlinear response to the excitation irradiance. We show that multicolored $\mathrm{UCL}$ from $\mathrm{NaYF}_{4}: \mathrm{Yb}^{3+}, \mathrm{Er}^{3+} \mathrm{aNaYF}_{4}: \mathrm{Yb}^{3+}, \mathrm{Nd}^{3+}$ and $\mathrm{NaYF}_{4}: \mathrm{Yb}^{3+}, \mathrm{Tm}^{3+} \mathrm{aNaYF}_{4}: \mathrm{Yb}^{3+}, \mathrm{Nd}^{3+}$ core/shell UCNPs can be increased by more than one order of magnitude under either 980 or $808 \mathrm{~nm}$ excitation, by simply placing a polymeric MLA onto the top of these samples. The observed typical green $(525 / 540 \mathrm{~nm})$ and red $(654 \mathrm{~nm}) \mathrm{UCL}$ bands from $\mathrm{Er}^{3+}$ and a blue $(450 / 475 \mathrm{~nm}) \mathrm{UCL}$ band from $\mathrm{Tm}^{3+}$ exhibit distinct enhancement factors due to their different multi-photon processes. Importantly, our ray tracing simulation reveals that the MLA is able to spatially confine the excitation light ( 980 and $808 \mathrm{~nm}$ ) by orders of magnitude, thus amplifying UCL by more than 225 -fold (the $450 \mathrm{~nm} \mathrm{UCL}$ band of $\mathrm{Tm}^{3+}$ ) at low excitation irradiance. The proposed MLA method has immediate ramifications for the improved performance of all types of UCNP-based devices, such as UCNP-enhanced dye sensitized solar cells demonstrated here.
\end{abstract}

\section{Introduction}

The infrared (IR) region, which contains almost half of the irradiated solar energy, has for a long time constituted the hardest part of the solar irradiation spectrum to be utilized in solar cells., ${ }^{1,2}$ This is because the involved photosensitizers such as dyes and perovskites typically have very limited response to IR light. ${ }^{3,4}$ Lanthanide-doped upconversion nanoparticles (UCNPs), which are able to convert the spectral energy in the IR range into shorter-wavelength IR, visible, or even the UV range, ${ }^{1,2,5-12}$ can provide a solution to circumvent the transmission loss in photovoltaic devices by converting two or more sub-band-gap photons into one above-band-gap photon, and potentially change the status of IR energy utilization of various types of solar cells.

\footnotetext{
${ }^{a}$ Department of Theoretical Chemistry and Biology, KTH Royal Institute of Technology, Stockholm, Sweden.E-mail: hagren@kth.se, haichun@kth.se ${ }^{b}$ School of Chemistry and Chemical Engineering, Harbin Institute of Technology, Harbin, P.R. China. E-mail: chenguanying@hit.edu.cn

${ }^{c}$ School of Optoelectronic Science and Engineering \& Collaborative Innovation Center of Suzhou Nano Science and Technology, Soochow University, Suzhou 215006, P.R. China

${ }^{d}$ College of Chemistry and Chemical Engineering, Henan University, Kaifeng, Henan 475004, P.R. China

$\dagger$ Electronic supplementary information (ESI) available. See DOI: 10.1039/ c9nr03105g
}

Recent advances in upconversion nanochemistry have led to a ready access to high-quality UCNPs with a high luminescence quantum efficiency up to $19 \%$ (our previous worldrecord results) and above. ${ }^{13,14}$ However, such a high quantum efficiency of UCNPs is concealed by their relatively high excitation-intensity threshold, typically well above $1 \mathrm{~W} \mathrm{~cm}^{-2}$, which is much higher than that of IR light in the solar spectrum. As a result, when incorporated into solar cells, much of the light converting capacity of UCNPs is wasted due to the low excitation irradiance. ${ }^{15,16}$ This is fundamentally rooted in the multi-photon nature of UCL.

In view of the nonlinearity of UCNPs, we have, in our previous work, proposed a strategy of modulating the temporal distribution of delivered excitation photons to boost UCL. ${ }^{17,18}$ By employing quasi-CW excitation (i.e. pulsed excitation with a $\mu \mathrm{s}-\mathrm{ms}$ pulse width), multi-photon UCL can be easily enhanced by orders of magnitude relative to that under equivalent $\mathrm{CW}$ excitation with identical average excitation intensity. In this work, we explore a spatial version of excitation light modulation to evoke the prominent intrinsic photon conversion capacity of upconversion nanocrystals, which can be achieved only at high excitation intensity. Technically, we propose to utilize polymer-based microlens arrays (MLAs) to spatially modulate the excitation light. It turns out that the light concentration caused by the MLA can lead to significant UCL enhancement, readily higher than one order of magnitude. We 
performed ray tracing simulations, linking to the excitation light intensity response of nanocrystals, to seek optimal microlens configurations for the upconversion enhancement. The proposed strategy can overcome the high excitationintensity threshold of upconversion nanomaterials and bring about future breakthroughs in their energy applications to enhance the performance of solar cells in the IR range. It should be noted that our approach is general and can be combined with other strategies, chemical or photonic, of enhancing UCL.

\section{Experiment}

\section{Materials}

Yttrium(III) chloride hexahydrate $\left(\mathrm{YCl}_{3} \cdot 6 \mathrm{H}_{2} \mathrm{O}, 99.99 \%\right)$, neodymium(III) chloride hexahydrate $\left(\mathrm{NdCl}_{3} \cdot 6 \mathrm{H}_{2} \mathrm{O}, 99.99 \%\right)$, erbium(III) chloride hexahydrate $\left(\mathrm{TmCl}_{3} \cdot 6 \mathrm{H}_{2} \mathrm{O}, 99.99 \%\right)$, thulium(III) chloride hexahydrate $\left(\mathrm{TmCl}_{3} \cdot 6 \mathrm{H}_{2} \mathrm{O}, 99.99 \%\right)$, sodium hydroxide $(\mathrm{NaOH})$, ammonium fluoride $\left(\mathrm{NH}_{4} \mathrm{~F}\right)$, 1-octadecene (ODE), oleic acid (OA), ethanol, methanol, and cyclohexane were purchased from Sigma-Aldrich. All the chemicals were used without further purification.

\section{Nanoparticle syntheses}

Synthesis of core $\mathrm{NaYF}_{4}: 20 \% \mathrm{Yb}^{3+}, 2 \% \mathrm{Er}^{3+}$ nanoparticles. Upconversion nanoparticles were synthesized with $\mathrm{NaYF}_{4}$ as the host material using a method as previously reported. ${ }^{19}$ $\mathrm{YbCl}_{3} \cdot 6 \mathrm{H}_{2} \mathrm{O} \quad(0.2 \mathrm{mmol}), \mathrm{YCl}_{3} \cdot 6 \mathrm{H}_{2} \mathrm{O} \quad(0.78 \mathrm{mmol})$ and $\mathrm{ErCl}_{3} \cdot 6 \mathrm{H}_{2} \mathrm{O}(0.02 \mathrm{mmol})$ were mixed with $15 \mathrm{~mL}$ 1-octadecene (ODE) and $6 \mathrm{~mL}$ oleic acid (OA) and heated to $160{ }^{\circ} \mathrm{C}$ and maintained for $30 \mathrm{~min}$ under an argon atmosphere to form a homogeneous solution. After the precursor solution cooled down to room temperature, a methanol solution of $0.1 \mathrm{~g}$ $\mathrm{NaOH}$ and $0.148 \mathrm{~g} \mathrm{NH} \mathrm{NH}_{4} \mathrm{~F}$ was slowly added and the mixture was stirred for $5 \mathrm{~min}$. The mixture was heated to $80^{\circ} \mathrm{C}$ and maintained for $30 \mathrm{~min}$ to evaporate methanol. Subsequently the solution was degassed, quickly heated to $300{ }^{\circ} \mathrm{C}$, and kept for $60 \mathrm{~min}$, under the protection of an argon atmosphere. The mixture was then cooled and the nanoparticles were purified through centrifugation using ethanol and water. The supernatant was discarded, and the precipitate was finally suspended in cyclohexane.

Synthesis of core-shell $\mathrm{NaYF}_{4}: 20 \% \mathrm{Yb}^{3+}, 2 \% \mathrm{Er}^{3+} @ \mathrm{NaYF}_{4}: 20 \%$ $\mathbf{Y b}^{3+}, 30 \% \mathrm{Nd}^{3+}$ nanoparticles. $\mathrm{NaYF}_{4}: 20 \% \mathrm{Yb}^{3+}, 2 \% \mathrm{Er}^{3+}$ @ $\mathrm{NaYF}_{4}: 20 \% \mathrm{Yb}^{3+}, 30 \% \mathrm{Nd}^{3+}$ core-shell nanoparticles were synthesized by epitaxially growing a $\mathrm{NaYF}_{4}: 20 \% \mathrm{Yb}^{3+}, 30 \% \mathrm{Nd}^{3+}$ shell onto the as-prepared $\mathrm{NaYF}_{4}: 20 \% \mathrm{Yb}^{3+}, 2 \% \mathrm{Er}^{3+}$ core nanoparticles following a previously reported protocol. ${ }^{20}$ In a typical synthesis, $\mathrm{YbCl}_{3} \cdot 6 \mathrm{H}_{2} \mathrm{O}(0.1 \mathrm{mmol}), \mathrm{YCl}_{3} \cdot 6 \mathrm{H}_{2} \mathrm{O}(0.25 \mathrm{mmol})$, and $\mathrm{NdCl}_{3} \cdot 6 \mathrm{H}_{2} \mathrm{O}(0.15 \mathrm{mmol})$ were mixed with $15 \mathrm{~mL} \mathrm{ODE}$ and $6 \mathrm{~mL}$ OA in a $100 \mathrm{~mL}$ three-neck flask. The solution was heated to $150{ }^{\circ} \mathrm{C}$ and kept for $30 \mathrm{~min}$ to form a homogeneous solution, and then cooled down to room temperature. A suspension of the $\mathrm{NaYF}_{4}: 20 \% \mathrm{Yb}^{3+}, 2 \% \mathrm{Er}^{3+}$ core nanoparticles dispersed in cyclohexane was added to the flask. The solution was maintained at $110{ }^{\circ} \mathrm{C}$ to remove the cyclohexane solvent and then subsequently cooled down to room temperature. A methanol solution of $0.05 \mathrm{~g} \mathrm{NaOH}$ and $0.075 \mathrm{~g} \mathrm{NH}_{4} \mathrm{~F}$ in $10 \mathrm{~mL}$ methanol was added into the flask and stirred for $5 \mathrm{~min}$. Then the solution was heated to $80{ }^{\circ} \mathrm{C}$ to remove methanol. After methanol was evaporated, the solution was heated to $300{ }^{\circ} \mathrm{C}$ and incubated for $60 \mathrm{~min}$ under an argon atmosphere. The mixture was then cooled down to room temperature. The nanoparticles were precipitated with acetone, collected after centrifugation, then washed three times with ethanol/ water $(1: 1 \mathrm{v} / \mathrm{v})$ and finally dispersed in cyclohexane for subsequent use.

Synthesis of core $\mathrm{NaYF}_{4}: 20 \% \mathrm{Yb}^{3+}, 0.5 \% \mathrm{Tm}^{3+}$ nanoparticles. The synthesis of core $\mathrm{NaYF}_{4}: 20 \% \mathrm{Yb}^{3+}, 0.5 \% \mathrm{Tm}^{3+}$ nanoparticles was similar to that of core $\mathrm{NaYF}_{4}: 20 \% \mathrm{Yb}^{3+}, 2 \% \mathrm{Er}^{3+}$ nanoparticles, but the types of lanthanide chlorides and their amount were adjusted accordingly.

Synthesis of core-shell $\mathrm{NaYF}_{4}: 20 \% \mathrm{Yb}^{3+}, 0.5 \% \mathrm{Tm}^{3+}$ @ $\mathrm{NaYF}_{\mathbf{4}}: \mathbf{2 0} \% \mathrm{Yb}^{3+}, \mathbf{3 0} \% \mathrm{Nd}^{3+}$ nanoparticles. The synthesis process of core-shell $\mathrm{NaYF}_{4}: 20 \% \mathrm{Yb}^{3+}, 0.5 \% \mathrm{Tm}^{3+} @ \mathrm{NaYF}_{4}: 20 \% \mathrm{Yb}^{3+}, 30 \%$ $\mathrm{Nd}^{3+}$ nanoparticles was similar to that of core-shell $\mathrm{NaYF}_{4}: 20 \%$ $\mathrm{Yb}^{3+}, 2 \% \mathrm{Er}^{3+} @ \mathrm{NaYF}_{4}: 20 \% \mathrm{Yb}^{3+}, 30 \% \mathrm{Nd}^{3+}$ nanoparticles, but the types of lanthanide chlorides and their amount were adjusted accordingly.

\section{Characterization}

The structural and morphological characterization was performed on a transmission electron microscope (JEOL, JEM-1400). Luminescence spectra were recorded on an Edinburgh FS5 spectrophotometer equipped with 808 and $980 \mathrm{~nm}$ diode lasers. An optical microscope (Olympus CX23) and a surface profiler (Veeco, Dektek 150) were used to characterize the surface structure of the MLA. A solar simulator (Newport Oriel, LSC-100) and a computerized Keithley 2400 source meter were used for current density-voltage (JV) measurement.

\section{Investigation of upconversion luminescence enhancement by addition of MLA}

A suspension of UCNPs in cyclohexane $(500 \mu \mathrm{L})$ was added dropwise onto the conducting side of a piece of FTO glass and then dried in mild air at room temperature. A collimated beam of NIR excitation light was shone on the nanoparticles, and the generated emission light was collected by a fiber, placed at the rear side of the FTO glass slide, and then detected by a connected spectrometer. When a piece of MLA was applied, it was attached to the UCNP surface with the assistance of a thin layer of solvent (water, ethanol, methanol) between the MLA and the UCNP layer.

\section{Current density-voltage $(J V)$ measurement and characteristics}

All the measurements given below were performed on dye sensitized solar cells (DSSC) purchased from the 3GSolar, Ltd. The current density-voltage measurements were performed with a solar simulator (Newport, AM 1.5G, illumination 
at $0.1 \mathrm{~W} \mathrm{~cm}^{-2}$ ) and a computerized Keithley 2400 source meter. UCNPs in cyclohexane $\left(250 \mu \mathrm{L}, 10 \mathrm{mg} \mathrm{mL}^{-1}\right)$ were first added dropwise onto a thin cover slide $(1.5 \mathrm{~cm} \times 1.5 \mathrm{~cm}$, a thickness of $0.17 \mathrm{~mm}$ ) and then dried in air to form a UCNP layer. Current density-voltage $(J V)$ measurements were performed on a reference DSSC, a DSSC with a dry UCNP layer or a MLA placed on top, and a DSSC with both a dry UCNP layer and an MLA placed on top to compare the efficiency differences. For all $J V$ measurements, a black mask was placed on top of the whole DSSC setup to create a $1.0 \mathrm{~cm} \times 0.7 \mathrm{~cm}$ exposure area.

\section{Results and discussion}

\section{Motivation of using microlens arrays as spatial light modulators to enhance upconversion luminescence}

Due to the nonlinearity of UCL, its quantum yield (QY) is generally not constant, but increases with the excitation intensity (Fig. 1). ${ }^{15}$ For instance, for a standard two-photon UCL band, the QY, $\Phi_{2-p h}$, is scaled with the excitation intensity $\left(I_{\mathrm{ex}}\right)$ according to: ${ }^{15}$

$$
\Phi_{2-\mathrm{ph}}=\frac{\Phi_{\mathrm{s}} I_{\mathrm{ex}}}{I_{\mathrm{b}}+I_{\mathrm{ex}}}
$$

where $\Phi_{\mathrm{s}}$ is the maximum QY that the two-photon UCL can achieve, and $I_{\mathrm{b}}$ is the balancing excitation intensity, at which the QY reaches the half of the maximum QY. Thus, given the same dose of excitation photons, a higher excitation intensity would lead to a larger number of emission photons.

Noticing that UCNPs demand high excitation intensity to trigger their light-conversion capacity, we devoted our efforts to seeking suitable light concentrators. We realized that micrometer-sized lens arrays, among others, can be ideal candidates to improve the performance of UCNP-sensitized devices (Fig. 1), e.g. solar cells. MLAs can not only efficiently concentrate excitation light due to their small radius of curvature, but also be easily integrated into photonic devices. In

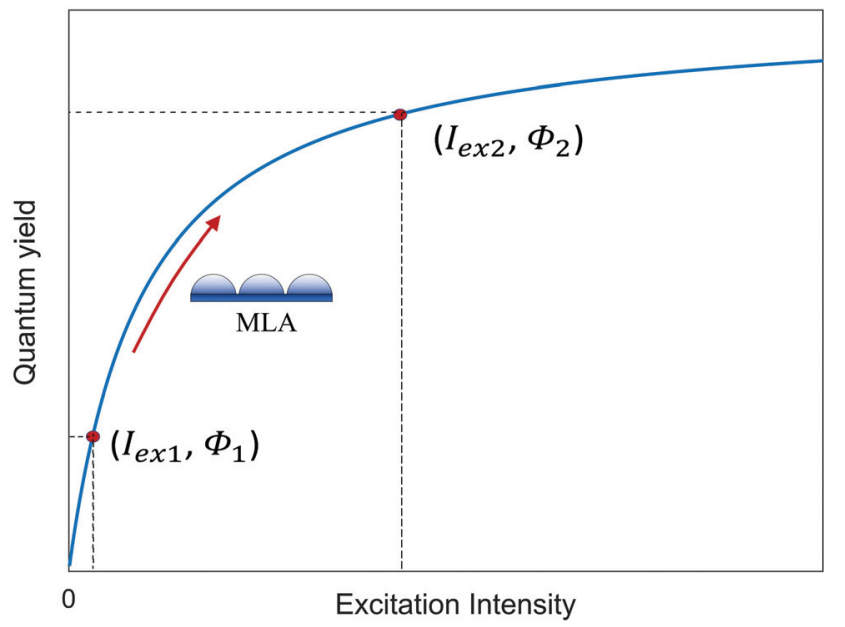

Fig. 1 Schematic illustration of a microlens array-enhanced quantum yield of upconversion luminescence due to its nonlinear response to excitation intensity. addition, the availability of relatively low-cost raw materials (e.g. polymer) and fabricating techniques potentially makes the cost affordable for large-scale production. In this work, we investigated the excitation-light modulation effect of MLAs on the luminescence intensity of UCNPs.

\section{Morphologies and optical properties of upconversion nanoparticles}

Core $\mathrm{NaYF}_{4}: 20 \% \mathrm{Yb}^{3+}, 2 \% \mathrm{Er}^{3+}$ and $\mathrm{NaYF}_{4}: 20 \% \mathrm{Yb}^{3+}, 0.5 \% \mathrm{Tm}^{3+}$ nanoparticles and the corresponding core-shell structured $\mathrm{NaYF}_{4}: 20 \% \mathrm{Yb}^{3+}, 2 \% \mathrm{Er}^{3+} @ 20 \% \mathrm{Yb}^{3+}, 30 \% \mathrm{Nd}^{3+}$ (denoted as YbEr@YbNd) and $\mathrm{NaYF}_{4}: 20 \% \mathrm{Yb}^{3+}, 0.5 \% \mathrm{Tm}^{3+} @ 20 \% \mathrm{Yb}^{3+}$, $30 \% \mathrm{Nd}^{3+}$ (denoted as YbTm@YbNd) nanoparticles were synthesized following previously reported protocols. ${ }^{19,20}$ The morphologies of the synthesized nanoparticles were characterized on a transmission electron microscope. The core $\mathrm{NaYF}_{4}: 20 \%$ $\mathrm{Yb}^{3+}, 2 \% \mathrm{Er}^{3+}$ nanoparticles have an average diameter of $\sim 30 \mathrm{~nm}$ (Fig. S1(a)†), and the core-shell YbEr@YbNd nanoparticles $\sim 40 \mathrm{~nm}$ (Fig. $\mathrm{S} 1(\mathrm{~b}) \dagger$ ). The $\mathrm{Tm}^{3+}$-doped core and core-shell nanoparticles have a similar average diameter to those of their $\mathrm{Er}^{3+}$-doped counterparts, $\sim 30 \mathrm{~nm}$ and $\sim 41 \mathrm{~nm}$, respectively (Fig. S1(c) and S1(d)†).

The UCL properties of the core-shell YbEr@YbNd and YbTm@YbNd nanoparticles were subsequently studied under continuous-wave (CW) 980 and $808 \mathrm{~nm}$ excitation. The YbEr@YbNd nanoparticles emitted relatively strong emission bands at 525/540 $\mathrm{nm}$ and $654 \mathrm{~nm}$ under both 980 and $808 \mathrm{~nm}$ excitation (Fig. S2(a)†), originating from the ${ }^{2} \mathrm{H}_{11 / 2} /{ }^{4} \mathrm{~S}_{3 / 2} \rightarrow$ ${ }^{4} \mathrm{I}_{15 / 2}$ and the ${ }^{4} \mathrm{~F}_{9 / 2} \rightarrow{ }^{4} \mathrm{I}_{15 / 2}$ transition of $\mathrm{Er}^{3+}$ ions, respectively. $^{21}$ The UCL intensity under $808 \mathrm{~nm}$ excitation was weaker than that under $980 \mathrm{~nm}$ excitation, as energy loss is caused in the indirect excitation approach of $808 \mathrm{~nm}$ light (via $\mathrm{Nd}^{3+} \rightarrow \mathrm{Yb}^{3+} \rightarrow \mathrm{Er}^{3+}$, which is equivalent to the direct excitation approach of $980 \mathrm{~nm}$ light (via $\left.\mathrm{Yb}^{3+} \rightarrow \mathrm{Er}^{3+}\right) .^{22} \mathrm{~A}$ much weaker emission band at $409 \mathrm{~nm}$, originating from the $\mathrm{Er}^{3+}$ ${ }^{2} \mathrm{H}_{9 / 2} \rightarrow{ }^{4} \mathrm{I}_{15 / 2}$ transition, ${ }^{23}$ was also detected in both excitation approaches. The excitation intensity response of the nanoparticles was then quantified for both 980 and $808 \mathrm{~nm}$ excitation, by varying the excitation power and recording the corresponding UCL spectra. The two-photon green emission bands at 525/540 nm exhibited a near quadratic dependence on the excitation intensity at low excitation intensity (2.93$36.29 \mathrm{~W} \mathrm{~cm}^{-2}, 23-285 \mathrm{~mW}$ with a beam diameter of $\sim 1.0 \mathrm{~mm}$ ) upon CW $980 \mathrm{~nm}$ excitation, featuring a slope efficiency of 1.5 (Fig. S2(b)†). Under the same conditions, the red band at $654 \mathrm{~nm}$ shows a steeper dependence on the excitation intensity, with a slope efficiency of 1.8. The three-photon blue band at $409 \mathrm{~nm}$ shows a slope efficiency of 2.2. The excitation intensity $(980 \mathrm{~nm})$ dependence of all these upconversion bands became less prominent with increasing excitation power, which can be ascribed to a saturation effect. ${ }^{15}$ These UCL bands of YbEr@YbNd nanoparticles exhibit a similar response to the intensity of $\mathrm{CW} 808 \mathrm{~nm}$ excitation light, but with a faster saturation trend (Fig. S2(c) $\dagger$ ).

The YbTm@YbNd nanoparticles emitted relatively strong emission bands at $650 \mathrm{~nm}, 475 \mathrm{~nm}$, and $450 \mathrm{~nm}$ (Fig. S2(d)†), 
originating from the transitions ${ }^{1} \mathrm{G}_{4} \rightarrow{ }^{3} \mathrm{~F}_{4},{ }^{1} \mathrm{G}_{4} \rightarrow{ }^{3} \mathrm{H}_{6}$, and ${ }^{1} \mathrm{D}_{2} \rightarrow{ }^{3} \mathrm{~F}_{4}$ of $\mathrm{Tm}^{3+}$, respectively. ${ }^{24}$ These emission bands all show a nonlinear dependence on the excitation intensity under both 980 and $808 \mathrm{~nm}$ excitations (Fig. S2(e) and S2(f)†).

\section{Upconversion luminescence enhancement by using a microlens array as an excitation light spatial modulator}

The MLA used in the experiments was made of polycarbonate (PC). The PC material has high transparency in the NIR range, up to $95 \%$ (Fig. S3 $\dagger$ ). Therefore, there is less than 5\% energy loss through the MLA structure. A scanning electron microscope (SEM) and an optical microscope were used to characterize the surface structure and profile of the MLA. As shown in Fig. 2(a), the side-view SEM image illustrates a well-defined MLA with a period of $51.02 \mu \mathrm{m}$. The height of the microlens is determined to be $16.47 \mu \mathrm{m}$ and the width is $47.08 \mu \mathrm{m}$, with a gap of $3.50 \mu \mathrm{m}$ between neighboring microlenses. The thickness of the slab part next to the curved layer is $75.00 \mu \mathrm{m}$. The top-view SEM image (Fig. 2(b)) and the optical microscopy image (Fig. 2(c)) well illustrate the periodic structure of the MLA.

The UCL spectra of the nanoparticle samples were recorded with and without the addition of the MLA into the optical path of the excitation beam (Fig. 2(d)). During the measurements, the MLA was attached to the nanoparticle layer with the help of a thin layer of ethanol solvent in between. Fig. 2(e) presents the result for the YbEr@YbNd nanoparticles under $980 \mathrm{~nm}$ excitation. Notably, the intensities of the green $(525 / 540 \mathrm{~nm})$ and red $(654 \mathrm{~nm})$ emission bands of $\mathrm{Er}^{3+}$ were enhanced by a factor of $\sim 3.6$ and $\sim 14.0$, respectively, after adding the MLA. The $\mathrm{Er}^{3+}$ blue emission band (409 nm), which was not detectable without the MLA at the applied excitation intensity (1.3 $\mathrm{W} \mathrm{cm}{ }^{-2}$ ), could be well detected after the addition of the MLA to modulate the excitation light. After adding the MLA there was a noticeable change in the emission color even to the naked eye during the experiments (inset of Fig. 2(e)). The modulation effect of the MLA when using $808 \mathrm{~nm}$ excitation light was also studied. As shown in Fig. 2(f), under $808 \mathrm{~nm}$ excitation, the green and red emission bands of $\mathrm{Er}^{3+}$ of YbEr@YbNd nanoparticles were enhanced by a factor of $\sim 3.7$ and 16.0 , respectively, after adding the MLA. Similar comparative studies were performed on the YbTm@YbNd nanoparticles. After the MLA was added into the optical path of the excitation beam (either 980 or $808 \mathrm{~nm}$ ), right in front of the nanoparticle layer, the intensities of the different UCL bands of $\mathrm{Tm}^{3+}$ were remarkably enhanced (Fig. $2(\mathrm{~g})$ and $(\mathrm{h})$ ). The strongest two-photon UCL band at $800 \mathrm{~nm}$ under $980 \mathrm{~nm}$ excitation was enhanced by a factor of 4.5 (Fig. $2(\mathrm{~g})$ ). The emission bands at $450 \mathrm{~nm}, 475 \mathrm{~nm}$ and $650 \mathrm{~nm}$ of $\mathrm{Tm}^{3+}$ became very significant after adding the MLA, with an enhancement factor of 108,45 , and 45 , respectively, compared to what was observed without the MLA (Fig. 2(g)). A similar upconversion enhancement effect of the MLA was also observed when using $808 \mathrm{~nm}$ excitation (Fig. 2(h)). Particularly, the $450 \mathrm{~nm}$ UCL band was amplified by up to 135 -fold (Fig. 2(h)) by the addition of the MLA. These results prove the excitation light modulation effect of the MLA. The observed enhancement factors for different UCL bands under excitation of different wavelengths correlate well with the excitation power response of the upconversion materials shown in Fig. S2. $\dagger$ Since the UCL intensity $\left(I_{\mathrm{f}}\right)$ is proportional to the $n$th power of the excitation intensity $\left(I_{\mathrm{ex}}\right)$, i.e., $I_{\mathrm{f}} \propto I_{\mathrm{ex}}^{n}$, the emission enhancement factor would be related to the slope factor $n$, with a larger $n$ leading to a larger enhancement. The bigger enhancement factors of the red $(654 \mathrm{~nm})$ emission band relative to the green $(525 / 540 \mathrm{~nm})$ bands for the YbEr@YbNd nanoparticles under either 980 or $808 \mathrm{~nm}$ excitation (Fig. 2(e) and (f)) are consistent with its steeper excitation power dependence compared to the latter (Fig. S2(b) and (c)†). Similarly, for the YbTm@YbNd nanoparticles, the higher-order multiphoton UC emission band with a larger slope factor $n$ (Fig. S2(e) and $(f) \dagger)$ is associated with a bigger emission enhancement factor (Fig. $2(\mathrm{~g})$ and $(\mathrm{h})$ ). In addition, the slightly bigger enhancement factors under $808 \mathrm{~nm}$ excitation compared to those under $980 \mathrm{~nm}$ excitation of different emission bands can also be explained by the corresponding steeper excitation power dependence.

Due to the same reason, UCL bands should show a decreasing enhancement factor with increasing incident excitation intensity, since they would exhibit gradual saturation, featuring an elevated excitation power dependence, as evident in Fig. S2. $\dagger$ We quantified the enhancement factors of different emission bands of the YbEr@YbNd and YbTm@YbNd nanoparticles under $808 \mathrm{~nm}$ excitation with different intensities, and the results confirmed our prediction (Fig. 3(a) and (b)). It is worth mentioning that the enhancement factor for the $450 \mathrm{~nm}$ band under $808 \mathrm{~nm}$ excitation reached $\sim 225$ at a low excitation intensity of $0.2 \mathrm{~W} \mathrm{~cm}^{-2}$ and dropped to 4.5 at an excitation intensity of $22.3 \mathrm{~W} \mathrm{~cm}^{-2}$ (Fig. 3(b)). An indication of the excitation intensity-dependent enhancement factor is that the use of MLA would remarkably boost the UCL intensity of UCNPs at excitation levels relevant to natural solar irradiation. We then measured the UC emission spectra of the YbEr@YbNd and YbTm@YbNd nanoparticles at low excitation irradiance $\left(0.1 \mathrm{~W} \mathrm{~cm}^{-2}\right)$ with and without the addition of the MLA. As shown in Fig. 3(c)-(f), the UCL enhancement is even more prominent than those shown in Fig. 3(a) and (b). Due to the weakness of the UCL without addition of the MLA, we could not quantify concrete enhancement factors for most emission bands.

Due to the instrument limitation, we could not accurately control the distance between the MLA and the nanoparticle layer. We tried to vary this distance by replacing ethanol with other solvents as the interface medium, with the intention of making use of their different viscosities and surface tensions. The MLA modulation experiment on the YbEr@YbNd nanoparticles under $980 \mathrm{~nm}$ excitation was repeated. As shown in Fig. S4, $\uparrow$ the UCL was significantly enhanced for all of the tested solvents, including ethanol, water and methanol. With water, the emission was much less enhanced, while without using any solvent, it was difficult to achieve emission enhancement (data not shown). 
(a)

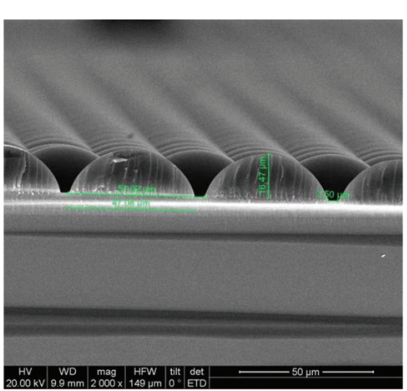

(c)

(e)

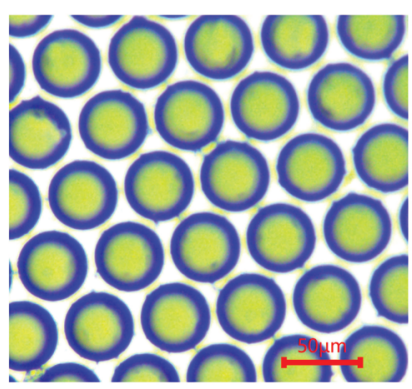

(b)

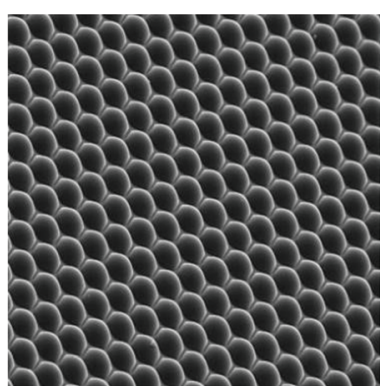

(d)

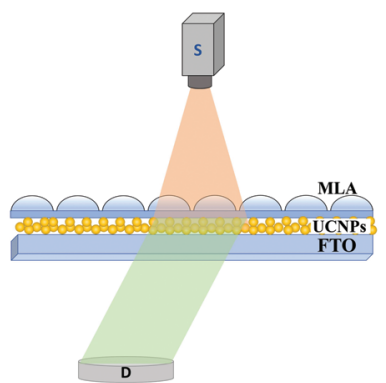

(f)

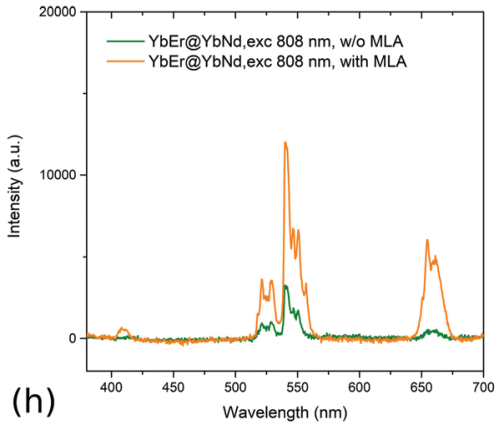

(h)

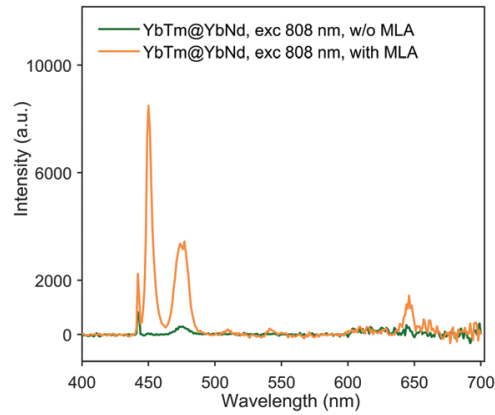

Fig. 2 SEM images of (a) the MLA composed of polycarbonate material, side view, and (b) surface structure, top view. (c) Optical microscopy image of the MLA. (d) Schematic of the optical setup for the MLA enhancement effect study: D - detector; $S-$ source. UCL spectra of NaYF $4: 20 \% \mathrm{Yb}^{3+}$, $2 \% \mathrm{Er}^{3+} \mathrm{a} 20 \% \mathrm{Yb}^{3+}, 30 \% \mathrm{Nd}^{3+}$ nanoparticles under (e) $980 \mathrm{~nm}$ and (f) $808 \mathrm{~nm} \mathrm{CW}$ excitation with and without MLA light modulation (average excitation intensity: $1.3 \mathrm{~W} \mathrm{~cm}{ }^{-2}$ ). UCL spectra of $\mathrm{NaYF}_{4}: 20 \% \mathrm{Yb}^{3+}, 0.5 \% \mathrm{Tm}^{3+}\left(\mathrm{a} 20 \% \mathrm{Yb}^{3+}, 30 \% \mathrm{Nd}^{3+}\right.$ nanoparticles under (g) $980 \mathrm{~nm}$ and (h) $808 \mathrm{~nm}$ $\mathrm{CW}$ excitation with and without MLA light modulation (average excitation intensity: $1.3 \mathrm{~W} \mathrm{~cm}-2$ ).

Ray-tracing simulations of the excitation light modulation effect of the MLA

To better understand the excitation light spatial modulation effect of MLA, ray tracing simulations were performed using Zemax, a commercially available software. Physical dimensions obtained from the SEM characterization, as schematically depicted in Fig. 4(a), and the optical properties of PC were used in the simulations. The simulated excitation beam (at $980 \mathrm{~nm}$ ), which was collimated with a top-hat intensity distribution before the MLA, had a very small incident angle onto the MLA surface. Fig. 4(b) and (c) present the simulated excitation light intensity distributions before the MLA (plane D1 in Fig. 4(a)) and at the right edge of the MLA (plane D2 in Fig. 4(a)). As can be seen, the incident beam was periodically re-distributed by the units of the MLA, with the transmitted 
(a)
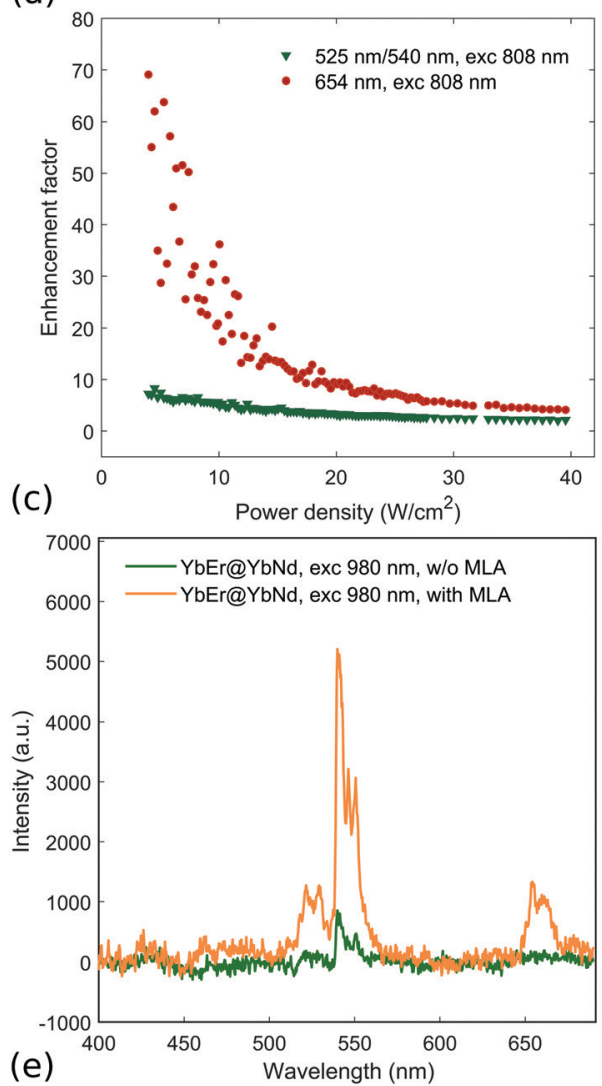

(e)

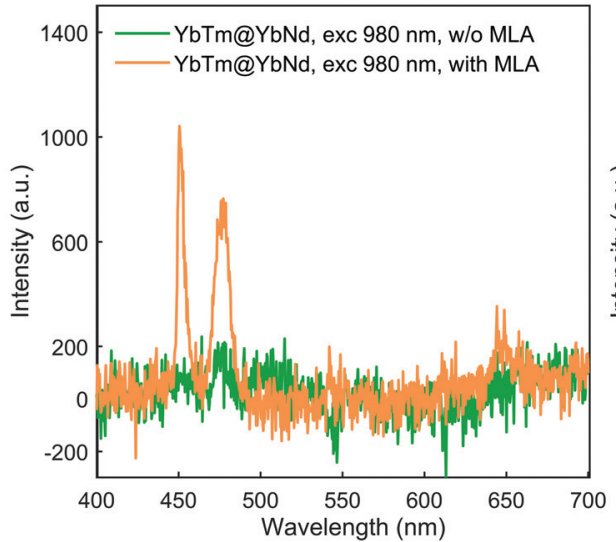

(b)
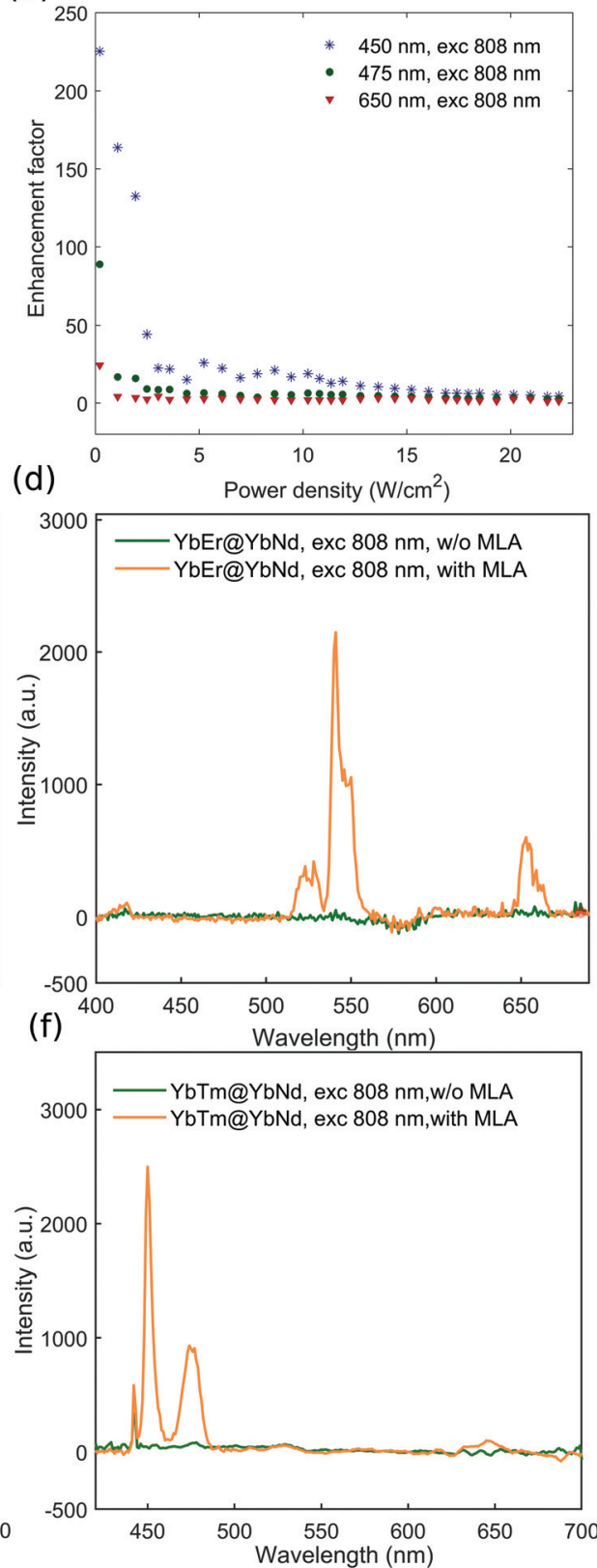

Fig. 3 Excitation intensity dependent enhancement factors induced by the addition of MLA of different emission bands of (a) $\mathrm{NaYF}_{4}: 20 \% \mathrm{Yb}^{3+}, 2 \%$ $\mathrm{Er}^{3+} \mathrm{a} 20 \% \mathrm{Yb}^{3+}, 30 \% \mathrm{Nd}^{3+}$ and (b) $\mathrm{NaYF}_{4}: 20 \% \mathrm{Yb}^{3+}, 0.5 \% \mathrm{Tm}^{3+} \mathrm{a} 20 \% \mathrm{Yb}^{3+}, 30 \% \mathrm{Nd}^{3+}$ nanoparticles under $808 \mathrm{~nm} \mathrm{CW}$ excitation. UCL spectra of $\mathrm{NaYF}_{4}: 20 \% \mathrm{Yb}^{3+}, 2 \% \mathrm{Er}^{3+} \mathrm{a} 20 \% \mathrm{Yb}^{3+}, 30 \% \mathrm{Nd}^{3+}$ nanoparticles under (c) $980 \mathrm{~nm}$ and (d) $808 \mathrm{~nm} \mathrm{CW}$ excitation with and without MLA light modulation (average excitation intensity: $0.1 \mathrm{~W} \mathrm{~cm}^{-2}$ ). UCL spectra of $\mathrm{NaYF}_{4}: 20 \% \mathrm{Yb}^{3+}, 0.5 \% \mathrm{Tm}^{3+}\left(\mathrm{a} 20 \% \mathrm{Yb}^{3+}, 30 \% \mathrm{Nd}^{3+}\right.$ nanoparticles under (e) $980 \mathrm{~nm}$ and (f) $808 \mathrm{~nm} \mathrm{CW}$ excitation with and without MLA light modulation (average excitation intensity: $0.1 \mathrm{~W} \mathrm{~cm}^{-2}$ ).

light concentrated at the focus of each unit (within the MLA). A line profile crossing the central row of the MLA at the right edge was extracted and compared with that on the incident plane prior to the MLA, as shown in Fig. 4(d). With the intensity for the incident top-hat beam set to $1.3 \mathrm{~W} \mathrm{~cm}^{-2}$, the peak intensity of the transmitted light after the very central unit was as high as $153 \mathrm{~W} \mathrm{~cm}^{-2}$. The excitation light modulation effect of the MLA on the resulting UCL was then estimated. The emission bands at 525/540 $\mathrm{nm}$ and $654 \mathrm{~nm}$ with the exci- tation-intensity response shown in Fig. S2(b) † were selected as the bands of interest. The UCL intensities integrated over the illuminated area when a nanoparticle layer was placed on the incidence plane $\left(I_{\mathrm{f}, \mathrm{i}}\right)$ and the MLA focusing plane $\left(I_{\mathrm{f}, \mathrm{o}}\right)$ were calculated by:

$$
I_{\mathrm{f}, \mathrm{i}(\mathrm{o})}=\iint_{0}^{\infty} \alpha R_{\mathrm{g}(\mathrm{r})}\left(I_{\mathrm{exc}, \mathrm{i}(\mathrm{o})}(x, y)\right) \mathrm{d} x \mathrm{~d} y
$$


(a)

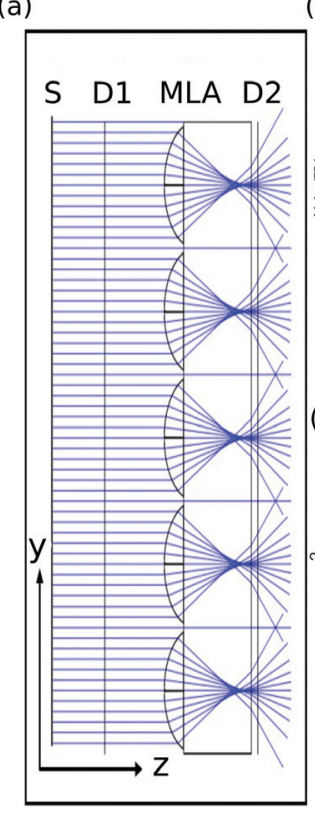

(b)

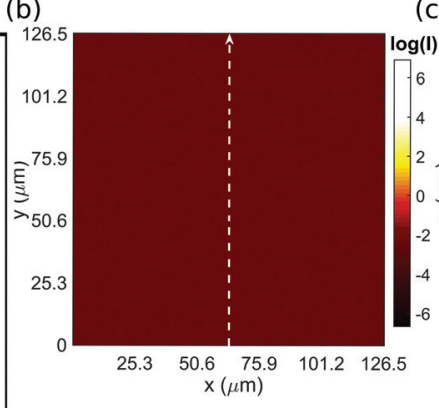

(d)

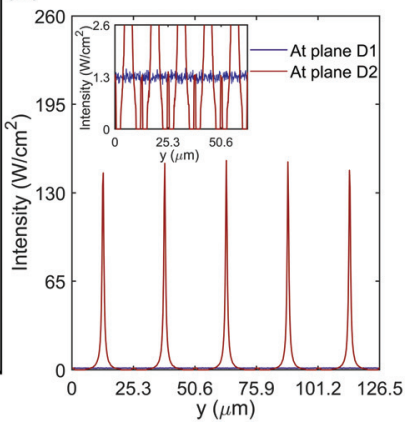

(c)

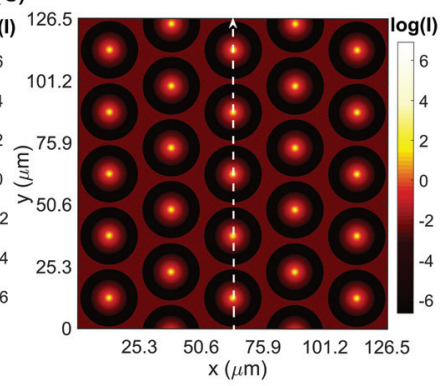

(e)

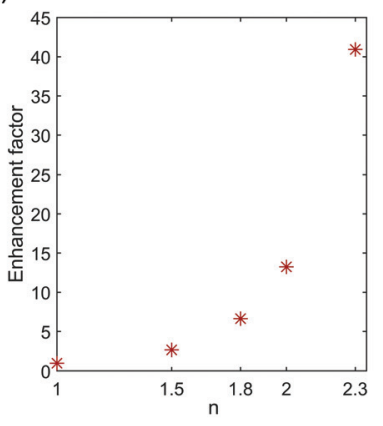

Fig. 4 (a) Geometry used in the ray-tracing simulations. Simulated excitation light intensity distribution (b) before and (c) at the right edge of the MLA. (d) Line profile analyses of excitation light intensity along the selected lines indicated by white lines in (b) and (c). (e) Calculated enhancement factors for upconversion emission bands with different slope factors.

where $\alpha$ is the scaling factor, and $R\left(I_{\text {exc }}\right)$ denotes the excitationintensity response of the band of interest shown in Fig. S2(b). $\dagger$ With the intensity of the incident beam set to $1.3 \mathrm{~W} \mathrm{~cm}^{-2}$, the calculated enhancement factors $\left(I_{\mathrm{f}, \mathrm{o}} / I_{\mathrm{f}, \mathrm{i}}\right)$ for the $525 / 540 \mathrm{~nm}$ and $654 \mathrm{~nm}$ bands are 6 and 44, respectively (Fig. 4(e)), which are in qualitative agreement with the obtained experimental results shown in Fig. 2(e). It should be noted that the calculated focusing plane of the MLA is $67.00 \mu \mathrm{m}$ after the curved part of the MLA $(6.50 \mu \mathrm{m}$ away from plane D2), located within the MLA, and thus maximum concentrated excitation light was not utilized in the experiments. This indicates a room to further increase the luminescence from the nanoparticle layer by optimizing the MLA structure to tune the position of the focusing plane, e.g., by adjusting the aperture, height, radius of curvature, and refractive index of the MLA.
Improved performance of UCNP-enhanced dye-sensitized solar cells by addition of a microlens array

To investigate the influence of the addition of MLA on UCNPenhanced dye-sensitized solar cells (DSSCs), four DSSCs were prepared, one reference cell (without UCNPs), the second and third incorporated with UCNPs or MLA respectively, and the fourth incorporated with both UCNPs and MLA. The device layout is shown in Fig. 5a. The prepared four cells were tested under direct AM1.5 G simulated sunlight irradiation (0.1 $\mathrm{W} \mathrm{cm}^{-2}$ ). As shown in Fig. 5b, the control DSSC shows a shortcircuit current density $\left(J_{\mathrm{sc}}\right)$ of $13.20 \mathrm{~mA} \mathrm{~cm}{ }^{-2}, V_{\mathrm{OC}}=0.698 \mathrm{~V}$, $\mathrm{FF}=58.19$ and $\eta=5.36 \%$. With the addition of UCNPs onto the photoanode, a $J_{\mathrm{sc}}$ of $13.50 \mathrm{~mA} \mathrm{~cm} \mathrm{~cm}^{-2}$ and a $\eta$ of $5.53 \%$ were achieved, featuring a $3.17 \%$ efficiency enhancement over the
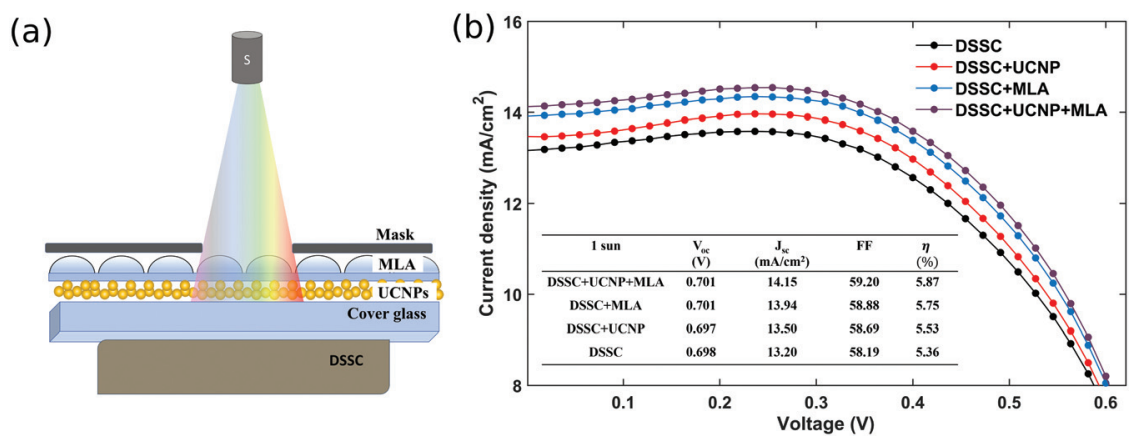

Fig. 5 (a) Schematic illustration of the configuration of UCNP- and/or MLA-enhanced DSSC. $S$ - source (b) the current density-voltage ( $J-V)$ characteristics of DSSC, DSSC + UCNP, DSSC + MLA and DSSC + UCNP + MLA under AM1.5 G light irradiation $\left(0.1 \mathrm{~W} \mathrm{~cm}{ }^{-2}\right)$. 
control cell. By adding a piece of MLA onto the photoanode, a $J_{\mathrm{sc}}$ of $13.94 \mathrm{~mA} \mathrm{~cm}^{-2}$ and a $\eta$ of $5.75 \%$ were achieved, featuring a $7.28 \%$ efficiency enhancement over the control cell. After the incorporation of both UCNPs and the MLA into the device, $J_{\mathrm{sc}}$ and $\eta$ were increased to $14.15 \mathrm{~mA} \mathrm{~cm}{ }^{-2}$ and $5.87 \%$, respectively, yielding a $6.15 \%$ overall efficiency enhancement over the solely UCNP-enhanced cell. Previously, MLAs have been employed to enhance the performance of solar cells, and it has been reported that MLAs are able to prolong the optical path of excitation light and enlarge the light collection angle of solar cells, as well as to concentrate light to facilitate the excitation process. ${ }^{25-29}$ In our case, the light concentrating effect should be dominant, considering the nonlinear response of UCNPs to excitation light intensity (Fig. S2 $\dagger$ ).

\section{Conclusions}

Lanthanide photon upconversion nanoparticles (UCNPs) generally exhibit a nonlinear response to excitation light, featuring a higher quantum efficiency at a higher excitation intensity. Thus, effective excitation light concentrators, whenever feasible, are preferred, to make better use of the photon-upconverting capacity of UCNPs. Here, we explored polymer microlens arrays (MLAs) as light concentrators for irradiating UCNPs and investigated their spatial light modulation effect on the resulting upconversion luminescence (UCL). It was found that a piece of MLA can potentially concentrate excitation light by orders of magnitude, subject to its structure and optical properties, and lead to a very significant enhancement of the UCL. MLAs can be easily incorporated into different types of UCNP-enhanced photonic devices, such as dye-sensitized solar cells, and bring further performance improvement in the near infrared range. A test on a dye-sensitized solar cell proved this contention, however, there is much room for optimizing a variety of parameters both for the solar cells and for the light concentrating upconverting layers to make the combined effects even more significant.

\section{Conflicts of interest}

H. ̊., H. L. and Q. L. have a patent application related to this work.

\section{Acknowledgements}

H. L. acknowledges a Starting Grant (2016-03804) from the Swedish Research Council (Vetenskapsrådet) and a grant from the ÅForsk Foundation (19-424). G. C. acknowledges the grants from the National Natural Science Foundation of China (51672061) and the Fundamental Research Funds for the Central Universities, China (HIT. BRETIV.201503 and AUGA5710052614). H. ̊. acknowledges a grant (2016-03619) from the Swedish Research Council (Vetenskapsrådet). Prof. Ying Yan at Soochow University is acknowledged for valuable discussions.

\section{References}

1 C. Yuan, G. Chen, L. Li, J. A. Damasco, Z. Ning, H. Xing, T. Zhang, L. Sun, H. Zeng, A. N. Cartwright, P. N. Prasad and H. Ågren, ACS Appl. Mater. Interfaces, 2014, 6, 1801818025.

2 C. Yuan, G. Chen, P. N. Prasad, T. Y. Ohulchanskyy, Z. Ning, H. Tian, L. Sun and H. Ågren, J. Mater. Chem., 2012, 22, 16709-16713.

3 C. Fei Guo, T. Sun, F. Cao, Q. Liu and Z. Ren, Light: Sci. Appl., 2014, 3, e161.

4 C. Xie, P. You, Z. Liu, L. Li and F. Yan, Light: Sci. Appl., 2017, 6, e17023.

5 S. Hao, Y. Shang, D. Li, H. Ågren, C.-H. Yang and G. Chen, Nanoscale, 2017, 9, 6711-6715.

6 H. Liu, K. Huang, R. R. Valiev, Q. Zhan, Y. Zhang and H. Ågren, Laser Photonics Rev., 2017, 12, 1700144.

7 G. Chen, H. Ågren, T. Y. Ohulchanskyy and P. N. Prasad, Chem. Soc. Rev., 2015, 44, 1680-1713.

8 M. K. G. Jayakumar, N. M. Idris and Y. Zhang, Proc. Natl. Acad. Sci. U. S. A., 2012, 109, 8483-8488.

9 K.-C. Liu, Z.-Y. Zhang, C.-X. Shan, Z.-Q. Feng, J.-S. Li, C.-L. Song, Y.-N. Bao, X.-H. Qi and B. Dong, Light: Sci. Appl., 2016, 5, e16136-e16136.

10 F. Wang, S. Wen, H. He, B. Wang, Z. Zhou, O. Shimoni and D. Jin, Light: Sci. Appl., 2018, 7, 18007.

11 R. Deng, F. Qin, R. Chen, W. Huang, M. Hong and X. Liu, Nat. Nanotechnol., 2015, 10, 237-242.

12 W.-P. Qin, Z.-Y. Liu, C.-N. Sin, C.-F. Wu, G.-S. Qin, Z. Chen and K.-Z. Zheng, Light: Sci. Appl., 2014, 3, e193e193.

13 B. Zhou, B. Shi, D. Jin and X. Liu, Nat. Nanotechnol., 2015, 10, 924-936.

14 G. Chen, J. Damasco, H. Qiu, W. Shao, T. Y. Ohulchanskyy, R. R. Valiev, X. Wu, G. Han, Y. Wang, C. Yang, H. Ågren and P. N. Prasad, Nano Lett., 2015, 15, 7400-7407.

15 H. Liu, C. T. Xu, D. Lindgren, H. Xie, D. Thomas, C. Gundlach and S. Andersson-Engels, Nanoscale, 2013, 5, 4770-4775.

16 J. He, W. Zheng, F. Ligmajer, C.-F. Chan, Z. Bao, K.-L. Wong, X. Chen, J. Hao, J. Dai, S.-F. Yu and D. Y. Lei, Light: Sci. Appl., 2017, 6, e16217-e16217.

17 H. Liu, C. T. Xu, G. Dumlupinar, O. B. Jensen, P. E. Andersen and S. Andersson-Engels, Nanoscale, 2013, 5, 10034-10040.

18 A. Bansal, H. Liu, M. K. G. Jayakumar, S. Andersson-Engels and Y. Zhang, Small, 2016, 12, 1732-1743.

19 Z. Li and Y. Zhang, Nanotechnology, 2008, 19, 345606345606.

20 H. S. Qian and Y. Zhang, Langmuir, 2008, 24, 1212312125.

21 J. Zhang, Z. Hao, J. Li, X. Zhang, Y. Luo and G. Pan, Light: Sci. Appl., 2015, 4, e239-e239.

22 J. Shen, G. Chen, A. M. Vu, W. Fan, O. S. Bilsel, C. C. Chang and G. Han, Adv. Opt. Mater., 2013, 1, 644-650. 
23 G. Chen, H. Liu, H. Liang, G. Somesfalean and Z. Zhang, Solid State Commun., 2008, 148, 96-100.

24 Q. Zhan, H. Liu, B. Wang, Q. Wu, R. Pu, C. Zhou, B. Huang, X. Peng, H. Ågren and S. He, Nat. Commun., 2017, 8, 1058-1058.

25 Y. Chen, M. Elshobaki, Z. Ye, J. M. Park, M. A. Noack, K. M. Ho and S. Chaudhary, Phys. Chem. Chem. Phys., 2013, 15, 4297-4302.
26 M. Nam, K. Kim, J. Lee, K.-K. Lee and S. S. Yang, Solar Energy, 2013, 91, 374-380.

27 A. Peer, R. Biswas, J. M. Park, R. Shinar and J. Shinar, Opt. Express, 2017, 25, 10704-10709.

28 M. Schmid and P. Manley, J. Photonics Energy, 2014, 5, 057003.

29 J. K. Tseng, Y. J. Chen, C. T. Pan, T. T. Wu and M. H. Chung, Solar Energy, 2011, 85, 2167-2178. 